\section{Possible Implication of Red Blood Cells in the Prothrombotic Risk in Early Rheumatoid Arthritis}

\section{To the Editor:}

Rheumatoid arthritis (RA) is an autoimmune inflammatory disease that can be considered as a prothrombotic state ${ }^{1}$. A great number of studies have investigated the possible role of reactive oxygen species (ROS) in the etiology and pathogenesis of this disease. The presence of large amounts of superoxide radicals and hydrogen peroxide produced by activated neutrophils has been reported in the synovial fluid of patients with RA. This may cause lipid peroxidation that yields a wide variety of end products, including malondialdehyde (MDA), a known marker of oxidative stress. These products are therefore transported from the synovial fluid to the blood circulation system ${ }^{2}$. Considering that elevated levels of MDA have been observed in the blood plasma of patients with $\mathrm{RA}^{2}$, the aim of this pilot study was to investigate whether the elevated levels of plasmatic MDA could be associated with a modification of the total antioxidant capacity (TAC) of blood plasma that is usually indicative of a "systemic" oxidative imbalance ${ }^{3}$. In addition, in view of their activity as redox effectors or scavengers ${ }^{4}$, as well as determinants of thrombus formation ${ }^{5}$, we evaluated red blood cell (RBC) features in terms of their redox state and lifespan marker molecules.

In our pilot study, 15 women with early RA (median age 44 yrs, range 20-56; median disease duration 24 weeks, range 12-60) and 15 age- and sex-matched healthy donors (HD) were included. Patients with RA were diagnosed according to the 2010 criteria of the American College of Rheumatology and European League Against Rheumatism ${ }^{6}$. Patients were excluded from the study if they had congenital heart disease, previous myocardial infarction, thrombotic events, renal or hepatic dysfunction, cancer, human immunodeficiency virus infection, or hepatitis. All patients (1) had active disease, defined as 28-joint Disease Activity Score $4.26 \pm$ 1.27 ; (2) had not yet been treated for RA; and (3) had no comorbidities, such as hypertension, hyperglycemia, hypercholesterolemia, or cardiovascular risk factors. Interestingly, no patients had anemia, and all showed high levels of C-reactive protein (CRP) and high values of erythrocyte sedimentation rate (ESR; Figure 1).

The nature and the purpose of our study were explained to all participants who gave their informed consent following the rules of good medical practice. This study was approved by the Institutional Review Board of "Sapienza" University of Rome, Italy, according to the principles outlined in the Declaration of Helsinki.

Plasma and RBC were isolated by centrifugation of whole blood ${ }^{7}$. RBC redox balance (levels of ROS and total thiol content), and expression of molecules involved in RBC senescence (CD47) and injury [Phosphatidylserine (PS) externalization] were evaluated as previously reported ${ }^{7}$. Expression of molecules responsible for RBC survival [survivin and extracellular signal-regulated kinase (ERK)] was evaluated by static (not shown) and flow cytometry using monoclonal antibodies (Santa Cruz Biotechnology and BD Biosciences). MDA and TAC were evaluated using commercially available ELISA immunoassay kits. The Mann-Whitney U test was used to compare quantitative variables in different groups. Flow cytometry data were statistically evaluated using the Kolmogorov-Smirnov nonparametric test $^{7}$ according to the Cell Quest Pro software guide (BD BioSciences).

In our study, we found that (1) as expected, plasmatic MDA levels were significantly higher $(\mathrm{p}<0.05)$ in patients with RA (median $2.62 \mu \mathrm{g} / \mathrm{ml}$, range 1.2-4.3) than in HD (median $1.73 \mu \mathrm{g} / \mathrm{ml}$, range 0.8-2.6), and (2) TAC of blood plasma was significantly reduced $(\mathrm{p}<0.005)$ in patients with RA (median $2.8 \mathrm{mM}$, range 2.2-5.4) compared to $\mathrm{HD}$ (median $21.35 \mathrm{mM}$, range 3.1-30.4). Interestingly, this does not seem to significantly interfere with the redox state of RBC and with externalization of PS. In fact, a mild production of ROS and a mild reduction of total thiol content have been detected in RBC from patients with RA. Similarly, no differences have been found in the percentage of RBC showing PS externalization (evaluated in
A

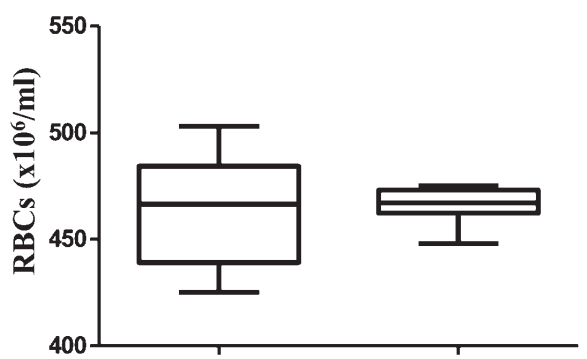

RA
B

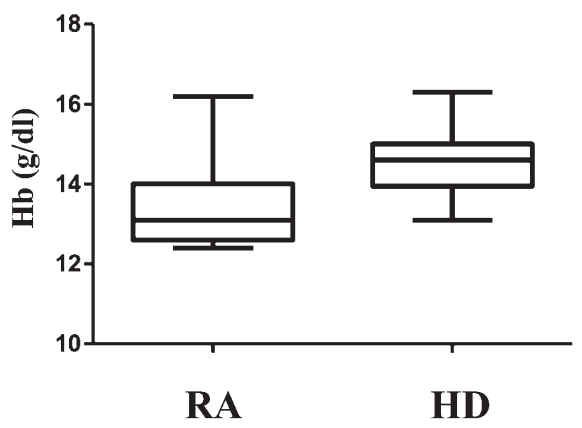

\section{D}

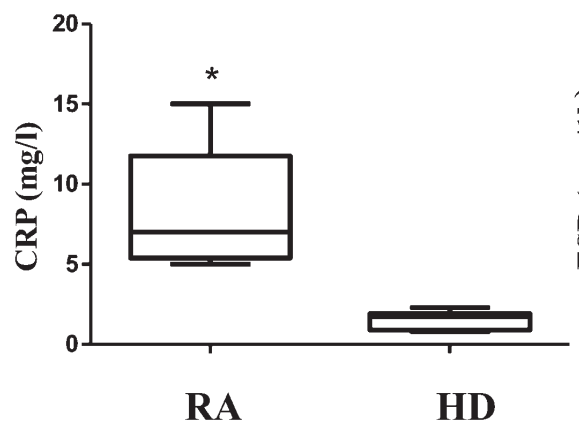

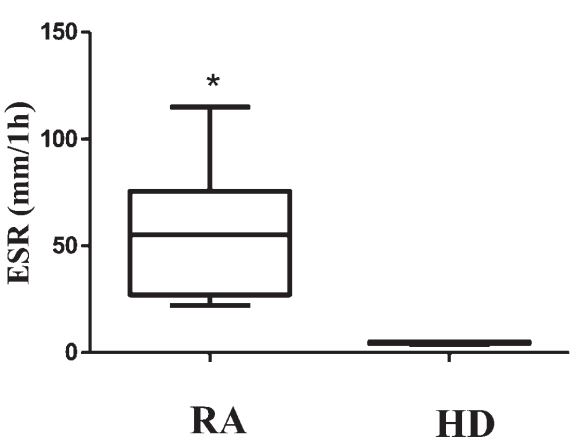

Figure 1. Evaluation of (A) RBC number, (B) Hb content, (C) CRP level, and (D) ESR value. Data are represented as box plots displaying medians, and 25th and 75th percentiles as boxes. Whiskers represented the smallest and the largest non-outlier values. $* \mathrm{p}<0.001$. RBC: red blood cells; Hb: hemoglobin; CRP: C-reactive protein; ESR: erythrocyte sedimentation rate. RA: rheumatoid arthritis; HD: healthy donors. 
terms of percentage of annexin V-positive RBC; Figure 2A, 2B, and 2C) and in the expression of CD47 (Figure 2D). Conversely, survivin ( $p<0.001$; Figure 2E) and phosphorylated ERK ( $<0.001$; Figure $2 \mathrm{~F})$ were significantly higher in RBC from patients with RA than in those from HD. CD47 is an integrin-associated protein, known as the thrombospondin receptor, which acts as a marker of self. Survivin is a member of the inhibitor of apoptotic protein family. ERK is a mitogen-activated protein kinase whose phosphorylation induces (1) upregulation of $\operatorname{survivin}^{8}$, and (2) increase of RBC adhesion to the endothelium ${ }^{9}$. Hence, our data, although obtained in a small cohort of patients, seem to indicate that in patients with RA, a mild alteration of intracellular redox state of RBC can occur. This is associated with activation of ERK signaling pathway and overexpression of survivin. This possibly leads to alterations of RBC homeostasis that, together with increased ESR and inflammatory protein $\mathrm{CRP}^{10}$, could alter blood flow ${ }^{1}$.

MANUELA DI FRANCO, MD, Department of Internal Medicine and Medical Specialties, Sapienza University of Rome; LUCREZIA GAMBARDELLA, MS, Department of Therapeutic Research and Medicine Evaluation, Istituto Superiore di Sanità; ANNA CHIARA DI LOLLO, MD, Department of Internal Medicine and Medical Specialties,

\section{A}

ROS

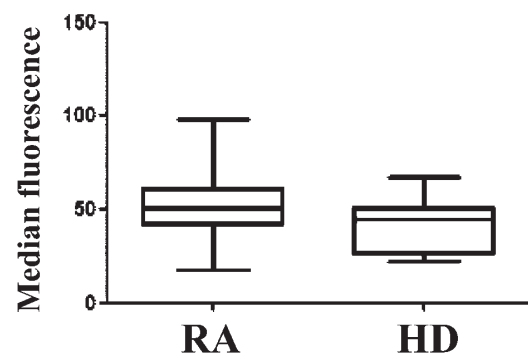

C
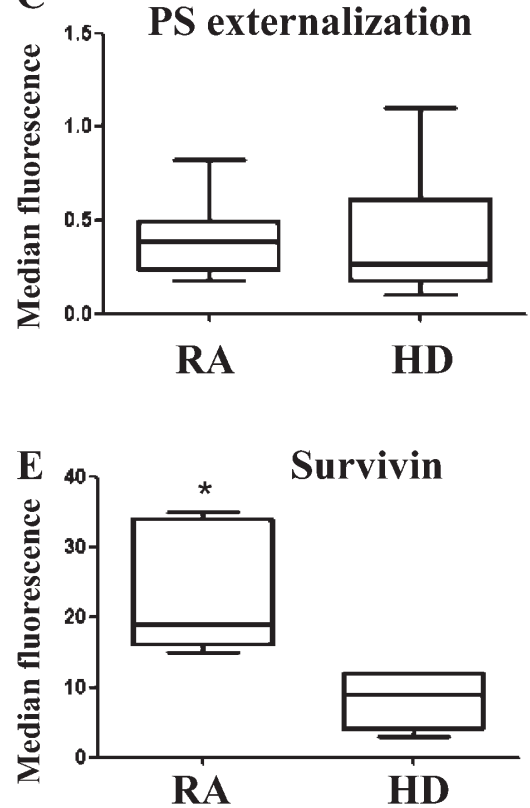

Sapienza University of Rome; WALTER MALORNI, PhD, Professor, Department of Therapeutic Research and Medicine Evaluation, Istituto Superiore di Sanità, and San Raffaele Pisana Institute; GUIDO

VALESINI, MD, Professor, Department of Internal Medicine and Medical Specialties, Sapienza University of Rome; ELISABETTA STRAFACE, $\mathrm{PhD}$, Doctor, Department of Therapeutic Research and Medicine Evaluation, Istituto Superiore di Sanità, Rome, Italy. Address correspondence to Dr. E. Straface, Istituto Superiore di Sanità, Department of Therapeutic Research and Medicine Evaluation, Section of Cell Aging and Gender Medicine, Viale Regina Elena 299 - 00161 Rome, Italy.

E-mail: elisabetta.straface@iss.it

\section{REFERENCES}

1. van den Oever IA, Sattar N, Nurmohamed MT. Thromboembolic and cardiovascular risk in rheumatoid arthritis: role of the haemostatic system. Ann Rheum Dis 2014;73:954-7.

2. Kamanli A, Naziroğlu M, Aydilek N, Hacievliyagil C. Plasma lipid peroxidation and antioxidant levels in patients with rheumatoid arthritis. Cell Biochem Funct 2004;22:53-7.

3. Straface E, Gambardella L, Metere A, Marchesi A, Palumbo G,

\section{B Reduced thiols}
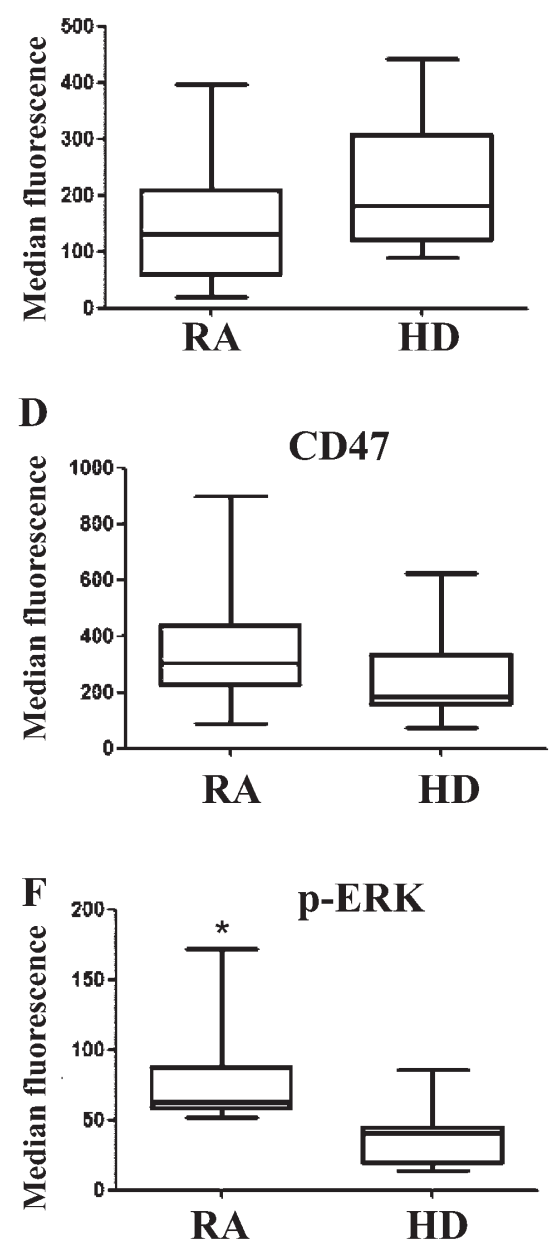

Figure 2. Flow cytometry analysis of (A) ROS production, (B) total thiol content, (C) PS externalization on RBC, (D) CD47 expression, (E) survivin expression, and (F) p-ERK expression. Data are represented as box plots displaying medians, and 25 th and 75 th percentiles as boxes. Whiskers represented the smallest and the largest non-outlier values. ${ }^{*} \mathrm{p}<0.001$. ROS: reactive oxygen species; PS: phosphatidylserine; RBC: red blood cells; p-ERK: phosphorylated extracellular signal-regulated kinase. 
Cortis E, et al. Oxidative stress and defective platelet apoptosis in naïve patients with Kawasaki disease. Biochem Biophys Res Commun 2010;392:426-30.

4. Minetti M, Agati L, Malorni W. The microenvironment can shift erythrocytes from a friendly to a harmful behavior: pathogenetic implications for vascular diseases. Cardiovasc Res 2007;75:21-8.

5. Aleman MM, Walton BL, Byrnes JR, Wolberg AS. Fibrinogen and red blood cells in venous thrombosis. Thromb Res 2014;133 Suppl 1:S38-40

6. Aletaha D, Neogi T, Silman AJ, Funovits J, Felson DT, Bingham CO, et al. 2010 rheumatoid arthritis classification criteria: an American College of Rheumatology/European League Against Rheumatism collaborative initiative. Ann Rheum Dis 2010; 69:1580-8.

7. Straface E, Marchesi A, Gambardella L, Metere A, Tarissi de Jacobis I, Viora M, et al. Does oxidative stress play a critical role in cardiovascular complications of Kawasaki disease? Antioxid Redox Signal 2012;17:1441-6.

8. Liu XY, Yao LL, Chen YJ, Tao BB, Yu YC, Bian WH, et al. Survivin is involved in the anti-apoptotic effect of edaravone in PC12 cells. Mol Cell Biochem 2009;327:21-8.

9. Zennadi R, Whalen EJ, Soderblom EJ, Alexander SC, Thompson JW, Dubois LG, et al. Erythrocyte plasma membrane-bound ERK1/2 activation promotes ICAM-4-mediated sickle red cell adhesion to endothelium. Blood 2012;119:1217-27.

10. Chung WS, Peng CL, Lin CL, Chang YJ, Chen YF, Chiang JY, et al Rheumatoid arthritis increases the risk of deep vein thrombosis and pulmonary thromboembolism: a nationwide cohort study. Ann Rheum Dis 2014;73:1774-80.

J Rheumatol 2015;42:7; doi:10.3899/jrheum.141396 